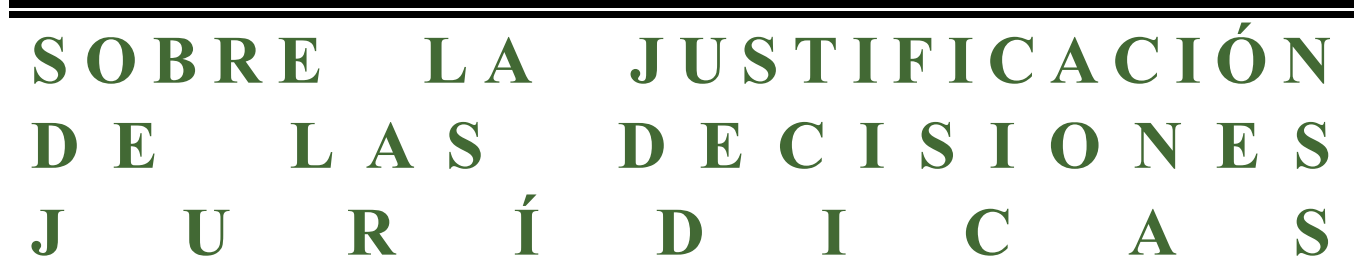




\section{LA TESIS DE LA ÚNICA \\ RESPUESTA CORRECTA \\ Y EL PRINCIPIO REGULATIVO \\ DEL RAZONAMIENTO JURÍDICO}

\section{Planteamiento}

os textos jurídicos son una parte especial del lenguaje. Por ello, todas las
dificultades que presenta la interpretación en general están necesariamente
presentes también en la interpretación jurídica. A menudo esto significa que los
textos jurídicos son ambiguos o que hay otros tipos de obscuridades en el texto.
Con el fin de aclarar los problemas que provoca tal falta de claridad pueden tomarse como ejemplo los llamados casos-situaciones difíciles.

El texto jurídico L permite cuatro interpretaciones alternativas y no equivalentes $I_{1}$ -- $\mathrm{I}_{4}$. Llamémoslas alternativas semánticamente posibles. Estas interpretaciones pueden concordar con el uso ordinario del lenguaje o con una parte especial de éste («lenguaje técnico»). En principio, estos significados pueden descubrirse recurriendo solamente a los métodos de la interpretación no jurídica. Sin embargo, el intérprete, por ejemplo el juez, tiene que hacer también una elección entre las alternativas expuestas. Su decisión tiene que ser jurídica. En esta misma elección se usa un patrón de interpretación jurídica establecido. Supongamos ahora que dos de las alternativas semánticamente posibles han sido seleccionadas como jurídicamente posibles, por ejemplo, $\mathrm{I}_{1}$ e $\mathrm{I}_{2}$.

La elección final, la decisión jurídica propiamente dicha debe realizarse entre estas dos posibilidades. En el proceso, el juez tiene además la obligación de dar una sola respuesta a cada caso. Por otro lado, la comunidad jurídica también espera una solución así de la dogmática jurídica. La gente espera que los dogmáticos recomienden la solución «apropiada» para cada caso. Por lo tanto, el problema de la única respuesta correcta se plantea no sólo en el ámbito judicial, sino también en el de la ciencia jurídica. 


\section{El concepto de única respuesta correcta}

El concepto de única respuesta correcta es ambiguo. Al me nos hay dos versiones diferentes de esta noción.

\section{1. La versión fuerte}

Según esta versión, para cada caso concreto existe una respuesta correcta que, además, puede ser detectada. La respuesta está «oculta» en algún lugar dentro del ordenamiento jurídico, y la habilidad del juez consiste justamente en hacer explícito lo que está ya implícito. Este tipo de versión fuerte fue representada por las doctrinas extremas del Derecho natural racionalista y de la jurisprudencia de conceptos (Begriffsjurisprudenz).

\subsection{La versión débil}

Esta versión acepta la idea de que existe la respuesta correcta pero vacila a propósito de las posibilidades de descubrirla. La única respuesta correcta no puede detectarse siempre (quizá nunca). Esta interpretación de la noción ha sido representada por algunas doctrinas positivistas. Por ejemplo, en Finlandia los principales administrativistas están de acuerdo con esta postura.

Como ha señalado Jerzy Wróblewski, el fundamento de esta versión es principalmente ideológico: el sistema jurídico tiene que carecer de lagunas. Por otra parte, cada juez o dogmático debería tener como guía cierta meta (la respuesta correcta). Como seres humanos que somos, tal vez no tengamos éxito a la hora de encontrar la respuesta correcta, pero a pesar de todo ella

está «allí». En otro caso -alguien podría decir-, toda toma de decisión jurídica sería arbitraria.

Hay, sin embargo, muchos problemas entrelazados con esta versión. Desde el punto de vista filosófico, uno de los más interesantes es el llamado dilema falibilístico: nunca se puede saber si la única respuesta correcta está disponible o no. ¿Qué significa entonces al final el concepto?

\subsection{La postura crítica}

En lo que sigue intentaré argumentar en favor de una respuesta negativa: no puede haber respuestas correctas en el razo- 
namiento jurídico (tesis ontológica). Por consiguiente, tales respuestas tampoco pueden ser detectadas (tesis epistemológica y metodológica). De la «naturaleza» misma de la ambigüedad semántica se desprende que hay que abandonar tanto la versión fuerte como la débil.

\section{Principales características del razonamiento jurídico}

\section{1. Tres perspectivas}

El razonamiento jurídico -tanto la toma de decisión judicial como la interpretación doctrinal- puede abordarse por lo menos desde tres perspectivas diferentes. La descripción heurística (contexto de descubrimiento) da información sobre el procedimiento real del razonamiento jurídico (¿cómo es hallada la respuesta?). La explicación causal o intencional, por otro lado, responde a los porqué (¿por qué las cosas suceden así y así?). Finalmente, el aspecto justificatorio (contexto de justificación) arroja luz sobre la argumentación (sobre ¿cuáles son las razones?). En lo que sigue, me centraré sólo en la justificación y mi tarea será dar una formulación más exacta a la sentencia:

P: Sobre la base de los argumentos G, la interpretación apropiada para el texto jurídico L es I.

\section{2. ¿Por qué es importante la justificación?}

El sistema del Estado de Derecho garantiza, entre otras cosas, un máximo de certeza jurídica para las partes de un proceso. La certeza jurídica puede caracterizarse incluso como una expectativa jurídica primaria de los miembros de la sociedad. De acuerdo con su formulación clásica, certeza jurídica significa negación de la arbitrariedad. Los ciudadanos tienen que poder planificar su conducta, y ello sólo es posible sobre la base de una práctica judicial previsible. En otras palabras, la interpretación que presentan los jueces no puede ser meramente azarosa o estar basada en la irracionalidad, tiene que basarse siempre en el Derecho y sólo en él. Así, en el sistema del Estado de Derecho la certeza jurídica se realiza, v.g., por medio de (1) la división de poderes, (2) la igualdad formal entre los ciudadanos, (3) la separación entre el Derecho y la moral y (4) el modelo formalista de razonamiento jurídico (el silogismo).

En todos los llamados Estados de bienestar la idea original 
del Estado de Derecho ha ido perdiendo gradualmente su significación de única ideología jurídica. El núcleo ya no estriba tanto en la igualdad formal entre la gente como en la material. La meta del Estado de bienestar es garantizar la calidad de vida y, coherentemente con ello, proteger a la parte más débil de la relación social. Esto significa que en el moderno Estado de bienestares imposible una separación estricta entre Derecho y moral. Justicia, razonabilidad, equidad son conceptos clave del sistema del Estado del bienestar y, por tanto, necesariamente también son criterios para las decisiones jurídicas.

En resumen, en una sociedad moderna la certeza jurídica cubre dos elementos diferentes (a) en el razonamiento jurídico ha de evitarse la arbitrariedad (principio del Estado de Derecho) y (b) la decisión misma, el resultado final, debe ser apropiado. De acuerdo con el punto (b), las decisiones jurídicas deben estar de acuerdo no sólo con el Derecho (formal), sino que también tienen que satisfacer criterios de certeza axiológica (moral). Utilizando la terminología de Max Weber, en una sociedad moderna el Derecho está materializado.

En la sociedad, la decisión jurídica sólo puede ser aceptable a condición de que ambos conjuntos de criterios (a) y (b), sean satisfechos. El proceso de razonamiento debe ser racional y sus resultados deben satisfacer las demandas de justicia. Además, si una decisión no es aceptable tampoco puede ser legítima -en el sentido amplio de la palabra.

En toda sociedad, un alto grado de legitimidad es una propiedad natural del orden jurídico. El nivel de certeza jurídica es una de las medidas importantes para valorar la legitimidad. Cuanto más alto es el grado de certeza jurídica, tanto mayor es la confianza de la gente en las funciones del orden jurídico. Así, los tribunales tienen una responsabilidad social especial en maximizar la certeza jurídica. Solamente entonces es aceptable su función. Sin embargo, ¿cómo conseguirla?

Una posible respuesta es la siguiente. En los Estados modernos (del bienestar) los jueces, o los tribunales en general, ejercen su responsabilidad justificando las decisiones de una forma bien conocida. Esta y sólo ésta les garantiza la autoridad necesaria en su función. La simple referencia a los textos jurídicos o a otros materiales estrictamente autoritativos no es suficiente. La gente pide más, y plantea una cuestión adicional: ¿por qué? La única respuesta a aquella cuestión es usar argumentos apropiados (razones). Por eso, en todas las sociedades modernas se ha incrementado la importancia del razonamiento jurídico. 


\subsection{Sobre las razones apropiadas}

Según Gunnar Bergholtz, este desarrollo está profundamente conectado con nuestra visión de la democracia. Esto es así debido al hecho de que una de las propiedades más importantes de una democracia madura es su carácter abierto. Ello hace posible el control externo de la toma de decisiones. Esto es verdad también por lo que concierne a la adjudicación. La independencia de los tribunales no significa que ellos estén completamente fuera del control democrático. La división de poderes garantiza la independencia de los tribunales sólo en relación a los otros centros de poder, especialmente frente al poder ejecutivo. Por otro lado, los tribunales de justicia son una parte de la sociedad y de su orden democrático. Los tribunales también deben estar sometidos a un control social ejercido por la gente. El único medio para este control es la exigencia de que los tribunales realmente argumenten sus decisiones.

La siguiente matriz da una visión general sobre los tipos de razones que pueden usarse como medios para este control:

\begin{tabular}{|l|c|c|}
\cline { 2 - 3 } \multicolumn{1}{c|}{} & Razones reales & Razones públicas \\
\hline $\begin{array}{l}\text { Razones jurídicamente } \\
\text { aceptables }\end{array}$ & NO & SÍ \\
\hline $\begin{array}{l}\text { Razones jurídicamente } \\
\text { inaceptables }\end{array}$ & NO & NO \\
\hline
\end{tabular}

«Razones reales» significa aquí una razón usada en la búsqueda de la solución (contexto de descubrimiento). Razones públicas, por su parte, se refiere a la justificación. Jurídicamente aceptables son las razones que muestran que la decisión está dentro del armazón del orden jurídico. Ellas «hacen» jurídica la decisión.

La matriz muestra que desde el punto de vista de la certeza jurídica sólo son importantes las razones jurídicamente aceptables y públicas porque sólo ellas permiten valorar la relevancia jurídica de la decisión. Si las razones permanecen ocultas y/o son inaceptables carecen de valor para el control público. Por ejemplo, si las decisiones están siempre razonadas sólo «entre

bastidores», entonces nunca se sabe por qué el uso del poder jurídico tiene tales o cuales contenidos. 


\section{La mejor justificación posible}

\subsection{La justificación interna y la externa}

\subsubsection{Sobre las fuentes del Derecho}

Las razones (argumentos) usados en la justificación jurídica se llaman fuentes del Derecho. En función de su historia y de sus condiciones sociales, cada cultura ha aceptado un conjunto de fuentes. Ellas pueden dividirse en grupos, por ejemplo, en fuentes autoritativas y substantivas. Las fuentes del Derecho autoritativas incluyen la ley, los trabajos preparatorios y los precedentes; por el contrario la expresión «razones substantivas» alude a, v. g., las opiniones doctrinales, los diversos tipos de razones prácticas (v. g., intereses), el Derecho comparado y los argumentos históricos. En ocasiones el concepto de «fuente del Derecho» se entiende en sentido estricto, es decir, como referido solamente a las razones autoritativas. En la tradición escandinava, sin embargo, la doctrina de las fuentes del Derecho es bastante liberal y abarca tanto a las razones autoritativas como a las substantivas.

\subsubsection{La justificación interna}

En el razonamiento jurídico es importante no sólo el catálogo de fuentes del Derecho sino también la manera de usarlas. Desde este punto de vista, puede distinguirse entre justificación interna y externa. La primera se refiere a la estructura interna («lógica») del razonamiento. El modelo clásico de justificación interna es el llamado silogismo aristotélico (modus ponens). La primera premisa consiste en la «base normativa» de la decisión (premisa normativa), mientras que la segunda describe el hecho del caso (premisa fáctica). La forma lógica de la inferencia garantiza que la conclusión, la decisión normativa, se siga de las premisas.

Toda decisión jurídica puede ex post ser escrita en la forma de un silogismo con independencia de la forma en que ha sido alcanzada. La justificación interna es, en este sentido, independiente del contexto de descubrimiento. Sin embargo, especialmente en los casos difíciles, siempre se puede poner en cuestión las premisas de la inferencia. Por ejemplo: ¿por qué se optó por la premisa normativa $\mathrm{Na}$ en lugar de $\mathrm{Nb}$ ? En tal situación, la premisa misma debe ser objeto de argumentación. Esta es tarea de la justificación externa. 


\subsubsection{La justificación externa}

La respuesta a este porqué (externo) es posible darla a través de otro silogismo, es decir, un silogismo en el que la premisa problemática aparece como conclusión. Pensemos en un ejemplo de justificación externa:

SILOGISMO I: PR1: Si F1, entonces (debe ser) G1.

PR2: f es F1.

C: Si f, entonces (debe ser) G1.

SILOGISMO II: $\quad$ PRI: Si en los trabajos preparatorios se dice que el texto jurídico L ( $\mathrm{Si} \mathrm{F}$, entonces debe ser $\mathrm{G}$ ) significa « $\mathrm{Si}$ $\mathrm{F} 1$, entonces $\mathrm{G} 1 »$, la interpretación $\mathrm{L}$ debe ser «Si F1, entonces (debe ser) G1».

PR2: $\quad$ Los Trabajos preparatorios dicen «Si F1, entonces G1».

C: Si F1, entonces (debe ser) G1.

De esta manera, se pueden construir cadenas de silogismos que dan soporte argumentativo a la decisión (interpretación). Una rama del «árbol silogístico» versa sobre los trabajos preparatorios, las otras sobre las decisiones de los tribunales (precedentes), sobre las consecuencias prácticas de la decisión o, por ejemplo, sobre las opiniones doctrinales de la ciencia jurídica. El «árbol silogístico» puede entonces verse como sigue:

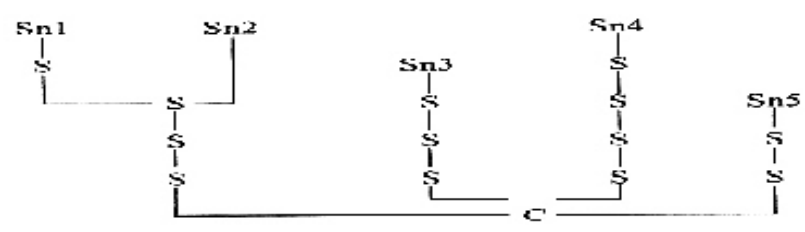


Aulis Aarnio

En un caso-situación difícil ninguna de las cadenas del silogismo da por sí misma soporte suficiente al resultado final, aunque ellas son necesarias por lo que se refiere a su fuerza justificatoria. El factor decisivo es la totalidad de los argumentos. En este sentido, la justificación externa es semejante a la construcción de un rompecabezas: el «mejor» resultado es la totalidad más homogénea ( $\mathrm{Sn} 1, \mathrm{Sn} 2, \mathrm{Sn} 3, \mathrm{Sn} 4, \mathrm{Sn} 5)$ que pueda construirse. Sin embargo, un rompecabezas normal y el razonamiento jurídico difieren entre sí en un aspecto muy importante. En un rompecabezas normal se sabe de antemano la figura que hay que construir. En el razonamiento jurídico «la figura» será desarrollada durante todo el juego y nadie sabe de antemano cuál es la totalidad de argumentos correcta.

Las premisas últimas de un árbol silogístico no son autoevidentes o empíricamente verdaderas. Son el resultado de sopesamientos y ponderaciones. Puede haber diferentes composiciones de argumentos, cada una de las cuales consiste en cadenas silogísticas internamente lógicas, pero de manera que las últimas premisas difieren de unas a otras. Lo decisivo aquí es el grado de coherencia del conjunto de premisas que se puede construir. La «medida última» es así la coherencia, no la correspondencia como en las afirmaciones empíricas.

Siguiendo con la metáfora del rompecabezas, la justificación externa ha sido denominada también diálogo. El intérprete $A$ presenta su postura $\mathrm{C} 1$ a otra persona, el destinatario $B$. $\mathrm{Al}$

principio, $A$ y $B$ están en desacuerdo entre sí y es necesario argumentar en favor y en contra de la conclusión, es decir, añadir nuevas premisas. En el curso de este razonamiento, $A$ y $B$ discuten sobre la importancia y el peso de sus argumentos. Al final, ambos pueden estar de acuerdo o en desacuerdo o llegar a un compromiso sobre la conclusión. En el último caso mencionado ellos alcanzan un consenso sobre la decisión «correcta».

\subsection{Persuasión versus convicción}

Entendida como un diálogo, la justificación jurídica que utiliza razones públicas no puede basarse en la manipulación. El principio de certeza jurídica no permite que la elección final entre las interpretaciones alternativas sea sólo el resultado de la persuasión o del uso del poder. Un buen diálogo justificatorio sigue siempre los estándares de la argumentación racional. Su meta es convencer a la otra parte, no manipularla. ¿Por qué? Porque toda la idea de lo que es Derecho está profundamente entrelazada con nuestras expectativas relativas a la conducta 
predecible de las autoridades. La arbitrariedad y la toma de decisiones al azar son ajenos a la concepción europea sobre el Derecho y la justicia.

Esto no significa, sin embargo, que cada caso particular tenga una y sólo una respuesta correcta. Por el contrario, la idea de razonamiento jurídico racional sólo presupone que las decisiones están tan bien argumentadas como sea posible. Nuestra expectativa concierne a la «mejor justificación posible», no a soluciones absolutamente correctas. Por ello en lo que sigue me ocuparé de la noción de «mejor justificación posible».

\subsection{La justificación ideal}

La mejor justificación posible presupone que no hay factores ocasionales que perturben el procedimiento de razonamiento. John Searle y Jürgen Habermas hablan al respecto de situación ideal de habla. El criterio decisivo para esta clase de situación (ideal) es la libertad. Todo aquel que participa en la discusión es inmune frente a la manipulación, el recurso a la fuerza mental o física, a la persuasión, etc. Además, todos los participantes deben ser iguales en la discusión, deben tomar parte en ella voluntariamente y deben estar dispuestos a seguir los estándares del discurso racional.

En relación con esto, Jürgen Habermas usa la expresión «racionalidad comunicativa» que significa la racionalidad que guía el discurso en una situación ideal de habla. Utilizando una terminología más precisa se puede hablar de racionalidad-D (racionalidad discursiva) como algo opuesto a, v. g., la racionalidad lógica (racionalidad-L).

Este tipo de situación de habla es, naturalmente, sólo un ideal. Sin embargo, es un ideal importante, porque puede utilizarse como medida para la corrección del discurso jurídico. Si el discurso jurídico no cumple esos tipos de criterios, no satisface nuestra concepción del Derecho y la justicia.

Ronald Dworkin utiliza para sus propios fines teoréticos un fenómeno ideal similar, el super juez llamado Hércules J. Este super juez lo sabe todo, dispone de tiempo ilimitado para tomar la decisión, su información sobre el caso y sobre los argumentos relevantes es ilimitada, es incluso capaz de hacer las elecciones más difíciles y, esto es importante para la imparcialidad, es capaz de cambiar su rol con otros sin ningún límite. Resumiendo: Hércules J. es una persona ideal que opera en la situación ideal de habla que tiene como tarea tomar las «mejores» decisiones jurídicas «posibles». 


\section{De nuevo sobre la única respuesta correcta}

¿Es Hércules J. capaz de producir con seguridad una respuesta correcta para cada caso difícil? Al principio, Dworkin parece inclinarse por una respuesta afirmativa. Este es justamente el rol de Hércules J. Más tarde, la tesis de Dworkin se hace más débil, pero, con todo, se mantienen las tesis centrales de su teoría de la única respuesta correcta. Robert Alexy defiende también una tesis debilitada: Hércules J. puede ser incapaz de producir una respuesta correcta para cada caso singular, pero está bien mantener la idea misma de una única respuesta correcta. Esta respuesta es como una meta oculta de nuestra toma de decisiones, un presupuesto ideológico.

Todos estos puntos de vista están expuestos a la crítica. Una manera de formular el núcleo de la crítica es preguntar: ¿qué ocurre si hay dos Hércules J.? Ambos son ciertamente seres racionales pero ¿son capaces de resolver genuinos problemas axiológicos? Esto es importante, porque las elecciones finales en el razonamiento jurídico no descansan solamente sobre la racionalidad del procedimiento del razonamiento y las estructuras libres que aseguran la discusión ideal. El otro factor decisivo es el «in put», es decir, los datos que se introducen en la discusión. En fin, Hércules J. también está vinculado v. g. a los intereses que presenta o está obligado a presentar en el discurso. Hablando toscamente, ellos no abordan el problema desde el mismo -o incluso similar- punto de vista. Por consiguiente dos o más Hércules J. pueden alcanzar varias respuestas no equivalentes pero igualmente bien fundadas.

Si se pretende ahora que una de las respuestas dadas es «mejor» que las otras o que es la única correcta, hay que tener algún criterio para esta postura. Y además, hay que salir fuera de la discusión, lo que significa recurrir a un metaHércules $J$., y así sucesivamente. Cada intento de probar que una de las respuestas alternativas es la «correcta» conduce así a un regreso al infinito argumentativo, y ello está condenado necesariamente al fracaso.

Esto también puede expresarse refiriéndose al concepto de coherencia. Lo que es necesario para una mejor solución posible es un argumento material coherente. Si hay dos o más conjuntos de razones igualmente coherentes, entonces la comparación no es posible. Desde un punto de vista justificatorio, todas ellas son igualmente buenas. Por medios racionales no podemos hacer más, porque no hay argumentos disponibles.

Así, una persona que hable de una solución «correcta» en este tipo de situaciones, remite a argumentos que están «detrás» 
de las razones públicas. Es imposible continuar la discusión racional con ella. No hay medios para establecer contraargumentos. Por consiguiente, todos estos argumentos «ocultos» son elementos innecesarios (sobre los que operaría la Navaja de Occam) para la teoría de la respuesta correcta.

\section{El principio regulativo del razonamiento jurídico}

\section{1. El concepto de auditorio}

Si no hay respuestas correctas para los casos difíciles, la siguiente pregunta es natural: ¿Tampoco es posible hablar de «la mejor respuesta posible»? Esta cuestión nos lleva al problema del principio regulativo.

Todo aquel que argumenta en favor o en contra de un cierto punto de vista dirige su argumentación a otra persona. A este respecto, el discurso jurídico es, también, un diálogo o, en general, un procedimiento discursivo. En un caso difícil hay dos partes en el diálogo, el intérprete (A) y el destinatario (B). Siguiendo a Chaim Perelman, puede llamarse auditorio al destinatario. Este es el auditorio del intérprete.

Demos un paso más allá y definamos dos conceptos adicionales:

La comunidad jurídica I consiste en todos los juristas profesionales y demás personas que tratan o pueden tratar con asuntos jurídicos. Esta comunidad es, pues, un «auditorio concreto» que existe realmente en la práctica jurídica. En la realidad, la argumentación dirigida a la comunidad jurídica I incluye elementos persuasivos porque la gente no satisface los criterios de Hércules J. Somos demasiado humanos, demasiado débiles y demasiado proclives a manipular a los demás como para ser seres ideales. En nuestras discusiones, ocultamos toda suerte de factores azarosos.

La comunidad jurídica II abarca a todos los que se comprometen con las reglas y principios de la racionalidad-D. Este auditorio es, pues, necesariamente ideal. Por lo tanto, la comunidad jurídica II es una medida por medio de la cual se puede evaluar el razonamiento y, lo que es importante, criticarlo.

Si todos los que prestan atención al procedimiento de razonamiento aceptan los estándares de la racionalidad-D, lo único que los separa entre sí es la diferente base material. No obstante, la idea de razonamiento jurídico racional presupone que cada uno dispone de todas las fuentes materiales. Todos los partici- 
pantes pueden usar de la información dada sin ninguna limitación específica. ¿Cómo es posible entonces que en la comunidad jurídica II se llegue a más de una respuesta? La clave de esta cuestión está en los materiales mismos de la argumentación.

En el discurso jurídico, muchas de las elecciones decisivas tienen carga valorativa. La elección entre las fuentes del Derecho, así como el peso de fuentes individuales, están parcialmente basadas sobre valoraciones. A menudo se hace referencia a la inferencia por analogía o a contrario. Ambas están vinculadas con valores; el concepto de «semejanza» no es valorativamente neutral. Todas las clases de metas argumentativas están conectadas al código de valores del que toma la decisión. Finalmente, los valores (y los puntos de vista morales) pueden estar directamente implicados en el razonamiento jurídico, como es el caso de los motivos sociales para el aborto.

Se puede así estar de acuerdo con Jerzy Wróblewski quien en diferentes contextos ha enfatizado que las interpretaciones jurídicas se basan en tres tipos de argumentos: (1) fuente material de Derecho (2) directivas de razonamiento jurídico y (3) valores y valoraciones. Las conclusiones finales son el resultado de todos estos factores. Esto sigue siendo verdad también en la comunidad jurídica II. Personas que aun estando de acuerdo con todos los estándares de racionalidad-D tienen códigos de valores diferentes.

La comunidad jurídica II no es, pues, un auditorio universal en el sentido de Perelman. Es universal sólo en la medida en que presupone la racionalidad del discurso. Todos los miembros aceptan el universo de racionalidad. Veamos más de cerca esta comunidad.

\subsection{Elprincipio de la mayoría}

El intérprete $A$ presenta la solución justificada $\mathrm{R}$ para el problema jurídico $\mathrm{P}$ ante la comunidad jurídica II. Supongamos, además, que la justificación incluye un punto de vista $\mathrm{S}$ sobre un valor intrínseco V. Dado que todos los miembros de la comunidad jurídica II están comprometidos con los estándares de la racionalidad-D y dado que todos disponen de los materiales relevantes, la diferencia en la aceptabilidad de $\mathrm{R}$ depende solamente de la valoración del punto de vista $\mathrm{S}$.

Ahora es posible que sólo una parte de la comunidad jurídica II esté de acuerdo con $A$. Esta parte de la comunidad hace justamente la misma interpretación de V que A. La otra parte hace 
otra interpretación de V, digamos S1. Esta parte de la comunidad defiende, pues, la solución R1 ¿Bajo qué condiciones podemos comparar las soluciones R y R1? ¿Es eso posible? Sí y no.

En esta comunidad ideal no hay una medida común para la comparación. No hay respuestas correctas y ninguna de las respuestas es en términos generales la mejor posible. Hay dos soluciones igualmente bien fundadas ( $\mathrm{R}$ y R1). No obstante, en esta situación una solución puede ser más relevante que otra, y desde esta perspectiva es también la «mejor posible». ¿Qué significa esto?

Una respuesta prometedora parece ser esta: si la mayoría de la comunidad jurídica II está dispuesta a aceptar, digamos, la solución $\mathrm{R}$, esta solución es por el momento la «mejor posible». Esto no significa que los miembros de la comunidad jurídica II «voten» en favor o en contra de R. La mayoría simplemente muestra la mayor aceptación posible para la solución R. En otras palabras, la solución R sería aceptable para la mayoría si R fuera discutida en este auditorio. Desde el punto de vista social, la otra solución es, como propuesta minoritaria, «más débil» que $\mathrm{R}$.

La crítica ahora puede venir por la pregunta de si este tipo de principio mayoritario viola los derechos de las minorías. Este no es el caso. Consideremos los siguientes argumentos:

1) Primero, este modelo considera sólo el razonamiento jurídico en una situación ideal de habla. La protección de la minoría tendría significación en una comunidad jurídica real (concreta), es decir, en un auditorio donde todas las clases de constelaciones de poder juegan un papel. En una comunidad ideal, como en la comunidad jurídica II, el uso de poder no es un problema. Esto se sigue del concepto de situación ideal de habla. Los participantes se supone que son libres.

2) En el discurso racional todas las razones relativas a los asuntos son tenidas en cuenta. Así, la opinión minoritaria forma parte también del razonamiento.

3) Dado que cada miembro de la comunidad jurídica II está comprometido con los estándares de la racionalidad-D, todos tienen que aceptar necesariamente también el principio mayoritario. Una persona racional puede preguntar: ¿cuál podría ser el principio alternativo si el principio de la mayoría no fuera aceptado? ¿Una decisión por sorteo?

4) La última pregunta mencionada está conectada a otra: ¿por qué no contentarse con dos (o más) decisiones igualmente bien fundadas $\mathrm{y}$, en consecuencia, racionalmente aceptables, $v$. 
g., con R y R1? ¿Por qué es necesario comprometerse con el principio mayoritario?

Como se ha señalado anteriormente, la aceptación de más de una respuesta posible es teóricamente incluso necesaria. En primer lugar, la visión relativista, asumida en esta contribución, parte del presupuesto de que no hay respuestas correctas en el ordenamiento jurídico ex ante. En segundo lugar, todas las soluciones bien fundadas, R y R1, son «correctas» ex post, en el sentido de que ellas son las respuestas apropiadas dentro de un seguro armazón justificatorio. No se pueden añadir más argumentos. Se ha llegado al límite del razonamiento racional, donde es imposible ir «más allá» de R o R1 en orden a compararlos.

Sin embargo, la cooperación social presupone que la gente pueda estar de acuerdo con ciertas soluciones. Esto puede ilustrarse como sigue. En las figuras siguientes, el símbolo «H» significa «Hércules J.» en el sentido dworkiniano.

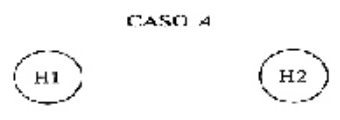

En este caso, la comunidad jurídica II consta sólo de dos miembros, H1 y H2. En ella se da un permanente desacuerdo entre puntos de vista, y este desacuerdo provoca incerteza sobre qué es correcto y qué es erróneo; y hay dos candidatos iguales para la solución «correcta». También en este caso, las autoridades necesitan una solución final para su puesta en práctica. Sin embargo, esta solución final no resuelve la tensión entre H1 y H2. No se puede saber si la solución final es correcta o no. Sólo es una de las alternativas encontradas. El problema del principio de la mayoría no puede plantearse porque no hay mayoría en la comunidad. La elección racional entre R y R1 sólo puede realizarse por sorteo.

CASO $B$

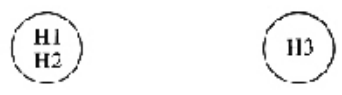


En este otro caso, H1 y H2 forman una mayoría en relación a H3. El desacuerdo acerca de las soluciones es el mismo que antes. Según nuestros presupuestos básicos esta comunidad jurídica es racional en cuanto a su naturaleza. A partir de este presupuesto resultan las siguientes tesis:

a) Todo $\mathrm{H}$ acepta los estándares de la racionalidad-D;

b) Todo $\mathrm{H}$ acepta la idea de cooperación social;

c) Todo $\mathrm{H}$ acepta el principio de que la cooperación social presupone la predictibilidad.

d) Todo $\mathrm{H}$ acepta el principio de que la predictibilidad no funciona si la solución se obtiene por sorteo.

Si se aceptan estos presupuestos, los mismos constituyen, al mismo tiempo, argumentos para otra tesis: cada caso difícil tendría una respuesta. Esto no significa que tal respuesta sea «la única correcta»; es solamente la respuesta más operativa a la luz de los presupuestos (a)-(d). «La más operativa», a su vez, significa que la mayoría de la gente en la comunidad puede estar de acuerdo con esta respuesta. En otras palabras, esta respuesta tiene el apoyo de la mayoría de una comunidad que no acepta decisiones por sorteo. A este respecto, puede decirse también que esta solución es por el momento «la mejor posible». Más tarde, la opinión minoritaria puede convertirse en opinión mayoritaria, lo que significa que las dinámicas sociales mismas cambian también las «mejores soluciones posibles» para el caso en discusión.

\section{Los contenidos del principio regulativo}

Tomando el análisis antes mencionado como punto de partida se puede proponer la siguiente directriz para el razonamiento jurídico:

En la decisión de un caso difícil se debe tratar de alcanzar una solución tal y una justificación tal que la mayoría de los miembros racionalmente pensantes de la comunidad jurídica pueda aceptar esa solución y esa justificación.

Este no es un manifiesto de ideología «Besser-Wisser». El principio regulativo no da ninguna autoridad a proclamar: tengo razón. Sólo trata de (1) incitar a la justificación racional al que toma la decisión y (2) prevenirle del objetivismo valorativo que es típico, $\mathrm{v}$. g., de Ronald Dworkin. La aceptabilidad por la ma- 
yoría de la comunidad jurídica II es más que lo que el juez o el dogmático pueden alcanzar en su razonamiento. Este tipo de aceptabilidad da la medida más objetiva también para la crítica.

Finalmente, la aceptabilidad como meta del razonamiento jurídico es suficiente para los seres humanos. Usando el principio regulativo se puede tratar de maximizar las expectativas de la certeza jurídica. Presuponer «una respuesta correcta» no nos ayuda a servir mejor a la sociedad. Lo que realmente necesitamos es justificación racional.

(Trad. de Josep Aguiló Regla)

\section{BIBLIOGRAFÍA}

Aarnio, Aulis, The Rational as Reasonable. Dordrecht, 1987.

Aarnio, Aulis; Alexy, Robert; Peczenik, Aleksander, The Foundation of Legal Reasoning.

Rechistheorie, 1981, págs. 133 y sigs., págs. 257 y sigs. y págs. 423 y sigs.

Alexy, Robert, Theorie der juristischen Argumentation, Frankfurt am Main, 1978.

—, Theorie der Grundrechte, Baden Baden, 1985.

Dworkin, Ronald, Taking Rights Seriously, 2. ${ }^{\mathrm{a}}$ ed. Londres, 1978.

Habermas, Jürgen, Theorie des kommunikativen Handelns: zur Kritik der funktionalistischen

Vernunft, I-II, Frankfurt am Main, 1981.

Peczenik, Aleksander, On Law and Reason, Dordrecht, 1989.

Perelman, Chaim, The Idea of Justice and the Problem of Argument, Londres, 1963.

Searle, J. R., Speech Acts: An Essay in the Philosophy of Language, Londres, 1969.

Summers, Robert S., The Types of Substantive Reasons: The Core of a Theory of Common Law Justification, Cornell Law Review (63), 1978, págs. 724 y sigs.

Wróblewski, Jerzy, Meaning and Truth in Judicial Decisión (ed. por Aulis Aarnio), 2.a ed., Tampere, 1983. 